\title{
O Tecnoestresse em Trabalhadores que Atuam com Tecnologia de Informação e Comunicação
}

Tecnostress of Workers on Information and

Communication Technologies

El Tecno-estrés en Trabajadores que Actúan con Tecnología de Información y Comunicación

Mary Sandra Carlotto \& Sheila Gonçalves Câmara

Universidade Luterana do Brasil 
Resumo: O tecnoestresse é um estado psicológico negativo relacionado com o uso de tecnologias de comunicação e informação (TIC) ou de ameaça de seu uso futuro. Esse fenômeno psicossocial é constituído de quatro dimensões: descrença, ansiedade, fadiga e ineficácia. O objetivo deste estudo foi identificar a existência de associação entre as dimensões do tecnoestresse e as variáveis demográficas, laborais e psicossociais em trabalhadores que utilizam TIC em 368 sujeitos de instituições de trabalho localizadas em Porto Alegre, RS e região metropolitana. Como instrumentos de coleta de dados, foram utilizados a Escala de Tecnoestresse - RED/TIC e um questionário para o levantamento de variáveis demográficas, laborais e psicossociais. Os resultados evidenciam que as mulheres apresentam médias mais elevadas nas dimensões de descrença e ansiedade. Foram identificadas associações principalmente no conjunto de variáveis psicossociais e, em menor proporção, nas variáveis laborais. Os resultados indicam a necessidade de intervenção em nível de prevenção, tendo em vista que o maior valor médio obtido foi na dimensão de fadiga, seguido pela de ansiedade.

Palavras-chave: Tecnoestresse. Tecnologia de comunicação e Informação. Prevenção. Fenômeno Psicossocial.

Abstract: Tecnoestress is a negative psychological condition related to the use of information and communication technology (ICT), or to the threat to their future use. This is a psychosocial phenomenon with four dimensions: disbelief, anxiety, fatigue and inefficiency. The aim of this study was to verify the existence of an association between the dimensions of tecnostress and demographic work and psychosocial variables in 368 subjects from work institutions located in Porto Alegre, RS, and the metropolitan region. For data collection, the instruments used were the Technostress - RED/ICT Scale and a questionnaire for the survey of demographic, work and psychosocial variables. Results show that women have higher averages in the dimensions of disbelief and anxiety. Associations identified mainly in the form set of psychosocial variables and a lesser proportion in the labor variables. The findings indicate the need of intervention at the prevention level due to the largest mean value at the fatigue dimension followed by the anxiety dimension.

Keywords: Tecnoestresse. Technology for communication and information. Prevention. Psychosocial phenomenon.

Resumen: El tecno-estrés es un estado psicológico negativo relacionado con el uso de tecnologías de comunicación e información (TIC) o de amenaza de su uso futuro. Ese fenómeno psicosocial está constituido de cuatro dimensiones: descreencia, ansiedad, fatiga e ineficacia. El objetivo de este estudio fue identificar la existencia de asociación entre las dimensiones del tecno-estrés y las variables demográficas, laborales y psicosociales en trabajadores que utilizan TIC en 368 sujetos de instituciones de trabajo localizadas en Porto Alegre, RS y región metropolitana. Como instrumentos de colecta de datos, fueron utilizados la Escala de Tecno-estrés - RED/TIC y un cuestionario para la pesquisa para recolección de datos de variables demográficas, laborales y psicosociales. Los resultados evidencian que las mujeres presentan medias más elevadas en las dimensiones de falta de creencia y ansiedad. Fueron identificadas asociaciones principalmente en el conjunto de variables psicosociales y, en menor proporción, en las variables laborales. Los resultados indican la necesidad de intervención en nivel de prevención, teniendo en vista que el mayor valor medio obtenido fue en la dimensión de fatiga, seguido por la de ansiedad.

Palabras clave: Tecno-estrés. Tecnología de comunicación e Información. Prevención. Fenómeno psicosocial.

As tecnologias de comunicação e informação (TIC), já é consenso, têm ocasionado inúmeras e profundas transformações em praticamente todas as áreas da atividade humana, envolvendo a economia, a política, a cultura, a própria organização do tecido social e das relações interpessoais. O novo contexto, no qual se insere a sociedade da informação, é construído com base nas tecnologias da comunicação e informação, fluindo através de velocidades e quantidades inimagináveis e representando profunda mudança na organização da sociedade e da economia. As atenções do mundo global e neoliberal direcionam-se para a importância e a necessidade da informação, sendo pertinentes questões acerca de sua produção, uso, armazenamento e recuperação. A informação passa a ser o principal fator de produção, capaz de interferir em qualquer contexto social (Souto, 2006). Verificase a emergência de um novo paradigma tecnoeconômico, também descrito como revolução industrial centrada em TIC, ou seja, no computador, no software, na microeletrônica, na internet e na telefonia 
móvel, que abrem novos territórios para a cultura digital. Mais que à informação, é ao processo de produção de conhecimento e conteúdo que remetem cada vez mais as estruturas tecnológicas digitais e interativas (Freeman \& Louçã, 2001).

Nos últimos anos, as mudanças tecnológicas vêm rearranjando as antigas corporações e orientando as novas empresas que nascem na era da informação, com o intuito de manter o seu longo exercício, em que essa nova constituição está formatada de tal maneira que se propõe a adequar-se às rápidas mudanças e a encarar de forma positiva fenômenos como globalização, mudanças políticas, sociais, econômicas e tecnológicas. A sociedade atual funciona galgada em conceitos de cunho tecnológico (Ferreira, 2006).

No contexto laboral contemporâneo, de acordo com Vale (1996) e Souto (2006), o trabalhador utiliza cada vez mais novas ferramentas para conseguir acompanhar e interagir com os diversos estágios de concepção e elaboração de produtos e serviços. Nesse sentido, novas características são exigidas, dando origem a um novo conceito e perfil de trabalhador. Neste, deve saber lidar com conceitos, processar e interpretar dados, reconhecer modelos e entender o processo produtivo como um todo, o que é radicalmente distinto do trabalhador fragmentado e preso ao pensamento mecanicista, que apenas executa seu trabalho. O novo trabalhador tem que se adaptar às novas linguagens, que se modificam constantemente. A área tecnológica é responsável por $80 \%$ dos postos de trabalho, e apenas $60 \%$ da força de trabalho se encaixa nessa realidade, recaindo sobre o trabalhador a obrigação de uma especialização e atualização frenéticas de conhecimento (Ferreira, 2006).

Segundo Pacheco, Ferreira Jr., Pereira e
Pereira Filho (2005), as organizações inserem tecnologia da informação (hardware) aos seus meios de produção, e, para tal, alteram substancialmente os métodos de produção (software), que, por sua vez, exigem alterações na forma de coordenação interna (orgware) e também um novo perfil de seu pessoal (peopleware). Esse é um fenômeno recente que, sob a ótica organizacional, altera as diretrizes do negócio, e, por conseguinte, todos os elementos de produção.

Assim, novas formas de trabalho e organizações se estruturam em torno das TIC, sendo as mais conhecidas o teletrabalho, as equipes virtuais e o e-comércio. Com os progressos tecnológicos dos últimos anos, o trabalho tem incorporado ao seu dia a dia pelo menos alguns elementos da interação virtual, envolvendo comunicação não presencial intermediada por e-mail, telefone, videoconferência, etc., sendo crescente o número de equipes compostas por pessoas que se comunicam quase que exclusivamente por meios eletrônicos (Bejarano, Pilatti, Scandelari \& Oliveira, 2006).

O avanço tecnológico, o surgimento da internet, da cibernética e, em consequência, da robótica, fez com que surgissem vários outros problemas relacionados ao mundo do trabalho (Ferreira, 2006). Essas inovações e mudanças tecnológicas se, por um lado, trazem benefícios econômicos para a organização e para muitos aspectos relacionados ao conteúdo do cargo do trabalhador, por outro, podem produzir problemas humanos e sociais que têm sido objeto de profundo debate devido às consequências para a saúde mental do trabalhador. A introdução de TIC no contexto de trabalho tem proporcionado efeitos positivos tanto quanto negativos para a qualidade de vida do trabalhador (Korunka, 2002).

A consequência direta para os trabalhadores, em razão dessa realidade, é que eles também 
De acordo com Michael (1998), com a inserção da tecnologia no ambiente de trabalho, mais pessoas estão propensas ao estresse tecnológico, denominado tecnoestresse. passam a depender das novas tecnologias, ou seja, direta e/ou indiretamente, para executar suas atividades laborais, Ihes é exigido não somente mais capacitação para se adequar aos novos meios mas também mais velocidade e mais dinamismo, em qualquer condição, como premissa organizacional. Então, nesse cenário de múltiplas exigências organizacionais de atualização e capacitação, o trabalhador é constantemente chamado a lidar com novas informações e cada vez mais se vê diante de inúmeras situações às quais precisa se adaptar. Seu trabalho requer maior exigência cognitiva e, por esse motivo, maior é a sobrecarga em seus processos mentais. Essa sobrecarga, causada pelos estímulos internos (necessidades e perspectivas pessoais) e externos (exigências dos sistemas produtivos e pressão social), é cada vez maior e mais recorrente. Diante das características da nova lógica de trabalho, é de se esperar que o estresse seja um fenômeno cada vez mais comum entre os trabalhadores, o que pode ser constatado pelos casos de desequilíbrios psicossomáticos e de doenças ocupacionais evidentes e/ou emergentes (Ferrreira, 2006; Pacheco et al., 2005).

De acordo com Michael (1998), com a inserção da tecnologia no ambiente de trabalho, mais pessoas estão propensas ao estresse tecnológico, denominado tecnoestresse. $\mathrm{O}$ conceito de tecnoestresse está diretamente relacionado aos efeitos psicossociais negativos do uso de TIC. Esse conceito foi abordado primeiramente por Brod (1984), que o definiu como uma enfermidade de adaptação causada pela falta de habilidade para tratar com as novas tecnologias de computadores de maneira saudável. Outras definições têm sido colocadas por outros autores como Weil e Rosen (1998), que o definem como qualquer impacto negativo sobre as atitudes, pensamentos, comportamentos ou fisiologia causados direta ou indiretamente pela utilização de tecnologia, não resultante apenas do contato das pessoas com o computador, mas também com qualquer forma de tecnologia, dos eletrodomésticos aos telefones celulares. Para Aida, Azlina e Balqis (2007), é uma doença moderna causada pela inabilidade de lidar com as novas tecnologias relacionadas ao uso de computador de forma saudável.

Tendo em vista a amplitude de tais definições, Salanova (2003) afirma que o tecnoestresse é um estado psicológico negativo relacionado com o uso de TIC ou com a ameaça de seu uso futuro. Esse estado está condicionado pela percepção de um desajuste entre as demandas e os recursos relacionados ao uso de TIC, que conduz a um alto nível de ativação psicofisiológica não prazerosa e leva ao desenvolvimento de atitudes negativas frente às $\mathrm{TIC}$.

Segundo Salanova, os principais fatores de risco do tecnoestresse são as altas demandas laborais com uso de TIC e a falta de recursos tecnológicos e sociais para lidar com elas. Demerouti, Bakker, Nachreiner e Schaufeli (2001) pontuam que altas demandas conjugadas a falta de recursos no trabalho (referentes à TIC) estão relacionadas ao aumento do tecnoestresse. Essa relação é modulada pela presença de recursos pessoais, tais como as próprias competências mentais e a autoeficácia relacionada com a tecnologia, dentre outros aspectos. Segundo os autores, as demandas laborais são os aspectos físicos, sociais e organizacionais do trabalho com TIC que requerem esforço e que estão associados a custos fisiológicos ou psicológicos. As principais demandas são: a sobrecarga de trabalho quantitativa (muitas coisas para serem realizadas em pouco tempo disponível) e a qualitativa (dificuldade em analisar e trabalhar com muitas informações); a rotina de trabalho (monotonia sem a exigência de esforço mental, isto é, atividade que requer pouca qualificação para sua execução); o conflito de papel (quando as demandas são percebidas como incompatíveis entre 
si, ou seja, instruções fornecidas por fontes diferentes), e o conflito trabalho/família. Os recursos laborais são os aspectos relacionados ao nível de autonomia do trabalho com as $\mathrm{TIC}$, com o feedback recebido e com o clima de apoio social por parte de chefias e colegas. Os recursos pessoais são as características do sujeito que funcionam como mediadoras do impacto negativo das altas demandas e da falta de recursos, principalmente as crenças sobre as próprias competências para fazer frente às novas tecnologias.

Com relação às consequências do tecnoestresse, segundo Salanova, Cifre e Matrín (1999), os resultados de investigações ainda são escassos, mas já é possível destacar, em nível individual, sintomas psicossomáticos tais como problemas de sono, dores de cabeça, dores musculares, transtornos gastrointestinais e, em nível organizacional, o absenteísmo e o baixo desempenho. Aida, Azlina e Balqis (2007) apontam dificuldades de concentração, irritabilidade e sentimentos de perda de controle, e estudo realizado por Staufer (1992) revelou que trabalhadores que possuíam atitude desfavorável em relação ao uso de tecnologia percebiam-na como ameaça e apresentavam queixas mais frequentes de dores de cabeça, problemas visuais e falta de concentração. Harper (2000) relata haver maior fracasso na resolução dos problemas organizacionais resultantes do alto nível de absenteísmo e de rotatividade de seus trabalhadores.

A investigação psicossocial tem estudado a problemática das consequências da introdução das TIC na saúde dos trabalhadores. Para Salanova, Cifre e Martín (1999), as mudanças geradas pelas novas tecnologias demandam ações de prevenção, a fim de evitar riscos e efeitos negativos para o trabalhador e, em consequência, para a organização.

Com base no modelo teórico constituído de três dimensões: 1) afetiva (ansiedade vs. fatiga), 2) atitudinal (atitude de descrença frente a tecnologias) e 3) cognitiva (crenças de ineficácia no uso de tecnologias) proposto por Salanova, Llorens, Cifre e Nogareda (2004), este estudo observacional analítico transversal (Grimes \& Shulz, 2002) procurou identificar a existência de associação entre as dimensões do tecnoestresse e as variáveis demográficas, laborais e psicossociais em trabalhadores que utilizam TIC em organizações situadas na região metropolitana de Porto Alegre, Rio Grande do Sul, no período de abril a setembro de 2007.

\section{Método}

\section{Amostra}

A escala foi aplicada em 368 sujeitos de instituições de trabalho localizadas em Porto Alegre, RS e região metropolitana. Os trabalhadores que constituem a amostra são, em sua maioria, mulheres $(56,5 \%)$, solteiros $(57,2 \%)$, sem filhos $(61,4 \%)$, com idade média de 30,68 anos (DP=9,95) e 6,91 anos $(\mathrm{DP}=5,52)$ de trabalho com TIC. Em relação à escolaridade, $68,1 \%$ possuem ou estão realizando curso superior. Quanto à função exercida, a maior parte dos sujeitos $(63,4 \%)$ trabalha em funções administrativas, e os demais participantes se distribuem nas funções de assistência técnica $(14,4 \%)$, médicos, engenheiros, advogados, relações públicas $(10,1 \%)$, vendedores $(5,8 \%)$, operadores de telemarketing $(4,9 \%)$ e professores $(1,4 \%)$. A maioria dos trabalhadores executa suas funções em empresas de grande e médio porte $(51,9 \%)$.

\section{Instrumentos}

Para o levantamento das variáveis demográficas (sexo, idade, escolaridade), laborais (função, tempo de trabalho, tempo de trabalho com TIC, tamanho da organização, número de pessoas que contata diariamente, quantidade de horas de uso de tecnologias) e psicossociais (pensar em mudar de profissão, percepção de que a profissão é estressante, percepção de que a utilização de TIC afeta a saúde física e mental, satisfação com o trabalho), foi elaborado um questionário especificamente para responder aos objetivos do estudo. 
A avaliação do tecnoestresse foi realizada com a Escala de Tecnoestresse - RED/TIC, desenvolvida pela equipe de investigadores da WONT - Work, Organization \& New Technologies - Departamento de Prevención Psicosocial da Universitat Jaume I de Castellón, e tem como base o modelo teórico da Psicologia social do trabalho, no campo da intervenção psicossocial e da saúde do trabalhador, que, por sua vez, está baseada no modelo RED (recursos, emoções/experiência, demandas), que entende o estresse como um processo interacional entre as demandas do ambiente de trabalho, e dos recursos ambientais e pessoais de que dispõe o sujeito para seu enfrentamento (Salanova, Llorens, Cifre, \& Nogareda, 2006). A RED/TIC - Escala de Tecnoestresse para Usuários de Tecnologias de Informação e Comunicação consiste de 16 questões, subdivididas em quatro dimensões: 1) descrença (itens 1, 2, 3, 4), 2); fadiga (itens 5, 6, 7, 8), 3); ansiedade (itens 9, 10, 11, 12), e 4) ineficácia (itens 13, 14, 15, 16). Todos os itens são avaliados em escala tipo Likert de sete pontos, variando de zero (nunca) a seis (sempre). O escore final de cada um dos atributos é dado pela média das respostas de seus itens, sendo que médias elevadas nas dimensões são indicadores de tecnoestresse. A escala validada na Espanha (Salanova et al., 2004) encontra-se disponível no site da WONT (http://www.wont.uji.es). Adaptação da escala (tradução para o português do Brasil e retrotradução para o espanhol) e estudo prévio das propriedades psicométricas (validade de conteúdo, validade de construto, confiabilidade) realizado pelas autoras da presente pesquisa identificaram que o modelo teórico se ajustou satisfatoriamente aos dados. Além de ter apresentado um valor de Quiquadrado estatisticamente significativo $\left(\mathrm{C}^{2}{ }_{\text {(98) }}\right.$ $=269,79, \mathrm{p}<0,000$ ), outros indicadores de bondade de ajuste dão suporte a este resultado: a razão $\mathrm{c}^{2} / \mathrm{gl}$ foi de 2,75 , com um GFI de 0,97 , e o RMSR, de 0,07 . O NNFI foi de $0,98, \mathrm{CFI}$ de 0,98 e PNFI $=0,79$. Todos os fatores apresentaram alfa de Cronbach superior a 0,70 . Assim, os resultados mostram que o RED-TIC oferece validade fatorial e consistência interna adequada para avaliar o tecnoestresse em profissionais brasileiros.

\section{Procedimentos}

Foram realizados os procedimentos éticos conforme Resolução no 196 do Conselho Nacional de Saúde (CNS) no que diz respeito à pesquisa com seres humanos. $\mathrm{O}$ estudo possui aprovação do Comitê de Ética em Pesquisa pela instituição de afiliação das autoras. Foi esclarecido aos sujeitos e diretores das instituições de trabalho tratar-se de uma pesquisa sem quaisquer efeitos avaliativos individuais e/ou institucionais e serem as respostas e os dados referentes aos resultados anônimos e confidenciais.

A coleta de dados, tanto no estudo piloto quanto no definitivo, foi realizada por estudantes de Psicologia, bolsistas de iniciação científica do Laboratório de Ensino e Pesquisa em Psicologia (LAEPPSI-ULBRA/Canoas), vinculados ao projeto. Os alunos foram treinados em dois encontros a fim de resguardar os procedimentos de padronização na aplicação. Após autorização, os instrumentos foram aplicados em salas de aulas e recolhidos logo após o preenchimento pelas pesquisadoras. Em uma segunda etapa, utilizou-se a técnica em cadeias (bola-deneve) (Biernacki \& Waldorf, 1981), em que os primeiros respondentes indicam outros, que, por sua vez, indicam outros, e assim sucessivamente. A seleção desses indivíduos, dentro de cada cadeia, respeitou os critérios de inclusão da amostra, o princípio da aleatoriedade e o voluntariado. Após indicação, alunos do Laboratório de Ensino e Pesquisa entraram em contato com os voluntários para convidá-los a participar do estudo.

\section{Resultados}

Os resultados obtidos indicam baixos índices nas dimensões de tecnoestresse, considerando uma escala de pontuação de 0 a 6, conforme a Tabela 1. Valor mais elevado foi encontrado na dimensão de fadiga, e o menor, em ineficácia. 
Tabela 1. Médias e desvio-padrão das dimensões de tecnoestresse.

\begin{tabular}{lllll}
\hline Dimensões & Min & Max & M & DP \\
\hline Descrença & 0,00 & 5,25 & 1,00 & 1,14 \\
Fadiga & 0,00 & 6,00 & 1,92 & 1,42 \\
Ansiedade & 0,00 & 6,00 & 1,57 & 1,22 \\
Ineficácia & 0,00 & 5,50 & 0,82 & 0,99 \\
\hline
\end{tabular}

Com relação às variáveis sexo e idade e escolaridade, mulheres apresentaram médias mais elevadas que homens nas dimensões descrença ( $M=1,12 ; \mathrm{H}=0,84 ; p=0,019)$ e ansiedade $(\mathrm{M}=1,71 ; \mathrm{H}=1,39 ; p=0,013)$. A idade ( DE $[r=0,46] ; \mathrm{FA}[r=-0,61] ; \mathrm{NA}[r=-0,001] ; \mathrm{IN}[r$ $=0,105]$ ) e a escolaridade ( DE $[r=-0,61]$; FA [ $r=0,017]$; NA [ $r=-0,098]$; IN $[r=-0,039]$ ) não apresentaram associação com nenhuma das dimensões do tecnoestresse.

Resultados obtidos através da prova de correlação de Pearson entre as dimensões de tecnoestresse e as variáveis laborais quantitativas evidenciam que a dimensão descrença se associa negativamente à frequência de realização de tarefas físicas (manipular, carregar) e mentais (processar informações, atenção) com TIC. A fadiga associou-se positivamente com as horas de trabalho realizadas na organização, com a utilização de e-mail e internet no trabalho. A ansiedade relacionou-se negativamente com o tempo de trabalho e tempo de trabalho, com TIC. Associação positiva foi identificada com a frequência de utilização de laptop e a participação em vídeo-conferências. A ineficácia associou-se negativamente com a realização de tarefas mentais. A percepção de que a trabalhar com TIC é estressante, de que sua utilização afeta a saúde física e emocional e também o pensamento de mudar de profissão relacionou-se positivamente com as quatro dimensões do tecnoestresse. Também ocorreu o mesmo resultado, no entanto, apresentando associação negativa com relação à satisfação com o uso de TIC no trabalho.

Tabela 2. Matriz de correção das dimensões de tecnoestresse e variáveis laborais quantitativas.

* Correlação significativa ao nível de $5 \%$.

** Correlação significativa ao nível de $1 \%$.

DE (descrença),

FA (fadiga), AN (ansiedade), IN (ineficácia)

\begin{tabular}{lclll}
\hline Variáveis & DE & FA & AN & IN \\
\hline Tempo de trabalho & $-0,023$ & $-0,099$ & $-0,114^{*}-0,007$ \\
Tempo de trabalho com TIC & $-0,039$ & $-0,006$ & $-0,111^{*}$ & $-0,019$ \\
Horas de trabalho com TIC na organização & $-0,061$ & $0,114^{*}$ & $-0,029$ & $-0,051$ \\
Horas de trabalho com TIC extra/organização & $-0,067$ & $-0,031$ & 0,034 & 0,057 \\
Pessoas que contata diariamente & $-0,024$ & 0,047 & $-0,045$ & $-0,075$ \\
Tamanho da organização & $-0,029$ & $-0,041$ & $-0,069$ & $-0,044$ \\
Horas de utilização de celular & $-0,080$ & 0,053 & $-0,002$ & $-0,080$ \\
Horas de utilização de computador & 0,014 & 0,006 & 0,025 & 0,041 \\
Horas de utilização de laptop & 0,083 & 0,057 & $0,160^{*}$ & 0,039 \\
Horas de utilização de e-mail & 0,041 & $0,139^{*}$ & $-0,022$ & $-0,043$ \\
Horas de utilização de internet & 0,003 & $0,129^{*}$ & $-0,015$ & $-0,032$ \\
Horas de utilização de vídeo-conferências & 0,103 & 0,147 & $0,418^{*}$ & 0,080 \\
Horas de utilização de chat & $-0,003$ & 0,108 & $-0,011$ & 0,003 \\
Tarefas físicas & $-0,118^{*}-0,102$ & $-0,039$ & $-0,044$ \\
Tarefas mentais & $-0,128^{*}$ & 0,027 & $-0,039$ & $-0,200^{*}$ \\
Tarefas sociais & $-0,043$ & 0,028 & $-0,024$ & $-0,057$ \\
TIC afeta a saúde física & $0,234^{* *} 0,438^{* *}$ & $0,235^{* *} 0,166^{* *}$ \\
TIC afeta a saúde emocional & $0,335^{* *} 0,485^{* *} 0,356^{* *} 0,270^{* *}$ \\
Percepção de estresse & $0,275^{* *} 0,420^{* *} 0,292^{* *} 0,239^{* *}$ \\
Satisfação no trabalho & $-0,328^{* *}-0,178^{* *}-0,230^{* *}-0,325^{* *}$ \\
Pensar em mudar de profisssão & $0,439^{* *} 0,404^{* *} 0,311^{* *} 0,292^{* *}$ \\
\hline
\end{tabular}




\section{Discussão}

Os resultados obtidos indicam baixos índices nas quatro dimensões que compõem o tecnoestresse, considerando as possibilidades de resposta da escala (0 a 6). Assim, de acordo com o modelo teórico e de avaliação, não se verifica sua ocorrência na amostra investigada. Esse resultado pode estar relacionado com o perfil da mesma, ou seja, os trabalhadores são jovens, com idade média de 30,68 anos ( $\mathrm{DP}=9,95)$, trabalham há pouco tempo com $\mathrm{TIC}, 6,91$ anos ( $\mathrm{DP}=5,52)$, sendo que o desvio-padrão indica grande variabilidade de respostas.

Com relação às variáveis demográficas, verifica-se que mulheres apresentaram médias mais elevadas que homens nas dimensões descrença e ansiedade. Estudo realizado por Zauchner, Korunka, Weiss e Kafka-Lützow (2000) encontrou resultado semelhante. Segundo os autores, essa situação ocorre porque as mulheres tendem a ocupar postos de menor qualificação e participação no trabalho.

Resultados obtidos através da matriz de correlação entre dimensões de tecnoestresse e variáveis laborais evidenciam que a descrença diminui na medida em que o trabalhador aumenta a frequência de realização de tarefas físicas (manipular, consertar) e mentais (processar informações, atenção). Pode-se pensar que, ao inteirarse tanto do sistema operacional como do processo, o trabalhador se torne mais confiante quanto à importância de seu uso para a obtenção de seus objetivos no trabalho.

A fadiga eleva-se na medida em que aumenta a carga horária de trabalho com TIC realizada na organização, assim como com a utilização de e-mail e internet. Esse resultado pode ser entendido a partir do modelo de demandas laborais de Demerouti, Bakker, Nachreiner e Schaufeli (2001). Para os autores, os aspectos sociais e organizacionais do trabalho com TIC requerem esforço, e estão associados a custos fisiológicos ou psicológicos. As principais demandas são a sobrecarga de trabalho quantitativa (muitas coisas para serem realizadas em pouco tempo disponível) e a qualitativa (dificuldade em analisar e trabalhar com muitas informações), situações características do trabalho realizado através de e-mails e da internet.

A ansiedade diminui com o aumento do tempo de experiência profissional e o tempo de trabalho com TIC. Pode-se pensar que, na medida em que o profissional adquire maior domínio das ferramentas de informação que necessita utilizar, diminui a ansiedade frente ao uso da TIC. Já a ansiedade aumenta com a frequência de utilização de laptop e da participação em vídeoconferências. O aumento da ansiedade pode estar relacionado à participação em vídeoconferências em função das temáticas e horários pré-determinados e ao uso do laptop, geralmente utilizado em trabalhos externos que terminam por gerar sobrecarga laboral e menor definição entre tempo de trabalho e tempo livre.

A ineficácia associou-se negativamente à realização de tarefas mentais, ou seja, quanto maior a execução de tarefas que utilizam capacidades mentais, maior é o sentimento de ineficácia para a utilização de TIC. Segundo Demerouti, Bakker, Nachreiner e Schaufeli (2001), a ineficácia é modulada pela presença de recursos pessoais, tais como as próprias competências mentais, a autoeficácia relacionada com a tecnologia, dentre outros aspectos. Os recursos pessoais são as características das pessoas que funcionam como mediadoras do impacto negativo das altas demandas e da falta de recursos. Dentre os principais recursos, existem características mais estáveis ao longo do tempo e também crenças sobre as próprias competências para fazer frente às novas tecnologias. 
Já a percepção de que trabalhar com TIC é estressante, de que sua utilização afeta a saúde física e emocional, pensar em mudar de profissão e a insatisfação no trabalho elevam as quatro dimensões do tecnoestresse. Essas variáveis, geralmente avaliadas como consequências do estresse ocupacional, podem se agravar com relação ao tecnoestresse.

\section{Conclusão}

Identificar o perfil de risco para o desenvolvimento do tecnoestresse é de fundamental importância para intervenções preventivas. O estudo aponta um quadro complexo de variáveis que podem prevenir ou ocasionar tecnoestresse na amostra investigada.

Deve-se ter cautela com relação aos resultados obtidos, uma vez que estes são decorrentes de organizações de trabalho localizadas em uma região específica do sul do Brasil, não sendo, portanto, passíveis de generalizações.
É importante considerar ainda o efeito do trabalhador sadio, questão peculiar em estudos transversais em epidemiologia ocupacional que, muitas vezes, exclui o possível doente (Mc Michael, 1976). Essa é uma situação que pode subestimar o tamanho dos riscos identificados, porque os mais afetados não conseguem manter-se no emprego, afastando-se geralmente por licenças para tratamento de saúde.

Certamente, há necessidade de aprofundamento dos resultados obtidos, uma vez que a literatura ainda é bastante restrita sobre esse recente fenômeno psicossocial. Assim, sugere-se a realização de novos estudos, com outros delineamentos e variáveis. Os resultados encontrados, porém, sinalizam a importância de intervenções que atuem sobre as variáveis laborais e psicossociais que influenciam o desenvolvimento do tecnoestresse em trabalhadores que utilizam TIC como ferramenta essencial no seu trabalho.

\footnotetext{
Mary Sandra Carlotto*

Doutora em Psicologia Social - Universidade de Santiago de Compostela, Espanha. Professora da graduação e pós-graduação da Universidade Luterana do Brasil, Canoas, RS - Brasil.
}

\section{Sheila Gonçalves Câmara}

Doutora em Psicologia pela Pontifícia Universidade Católica do Rio Grande do Sul, Porto Alegre, RS - Brasil.

Professora da graduação e pós-graduação da Universidade Luterana do Brasil, Canoas, RS - Brasil.

E-mail: scamara@via-rs.net

\section{* Endereço para envio de correspondência:}

Av. Mauá, 645, ap. 504, Centro - São Leopoldo, RS - Brasil - CEP 93110-320.

E-mail:mscarlotto@pesquisador.cnpq.br 


\section{Referências}

Aida, R. I. R. Z., Azlina, A. B., \& Balqis, N. S. (2007). Ergonomics and health aspects of work with computers. Heidelberg: Springer Berlin.

Amirkhan, J. H. (1994). Criterion validity of a coping measure. Journal of Personality Assessment, 62, 242-261.

Bejarano, V. C, Pilatti, L. A, Scandelari, L., \& Oliveira, A. C. (2006). Equipes virtuais - um estudo de caso na indústria têxtil norte-americana. Produção, 16(1), 161-170.

Biernarcki, P., \& Waldorf, D. (1981). Snowball sampling-problems and techniques of chain referral sampling. Sociological Methods and Research, 10, 141-163.

Brod, C. (1984). Technostress: The human cost of the computer revolution. Massachusetts: Reading Mass Addison-Wesley.

Demerouti, E., Bakker, A. B., Nachreiner, F., \& Schaufeli, W. B. (2001). The job demands-resources model of burnout. Journal of Applied Psychology, 86, 499-512.

Ferreira, A. P. C. (2006). Tecnologia de informação, controle e mundo do trabalho: pensar tecnologia na ótica do trabalhador. Revista Eletrônica de Ciências Sociais, 11, 14-24.

Freeman, C., \& Louçã, F. (2001). As time goes by: From the industrial revolutions to the information revolution. Nova York: Oxford University Press.

García-Jiménez, E., Gil-Flores, J., \& Rodríguez-Gomez, G. (2000). Cuadernos de estadística: Análisis factorial. Madrid: La Muralla.

Grimes, D. A., \& Shulz, K. F. (2002). An overview of clinical research: The lay of the land. The Lancet, 359, 57-61.

Harper, S. (2000). Managing technostress in UK Libraries: A realistic guide. Recuperado em 04 de agosto de 2006, de http://www.ariadne.ac.uk/issue25/technostress/intro.html

Korunka, C. (2002). Human-computer interaction and quality of working life: Organizacional aspects of implementing new technologies. In E. Chifre, M. Salanova \& Martínez, I. (Eds.), New information technology and work psychology: European trends towards fitting individual, job and organizational characteristics to new information technology (pp. 34-48). Castelló de la Plana: Publicaciones de la Universitat Jaume I.

Mc Michael, A. J. (1976). Standardized mortality ratios and the healthy worker effect: Scratching beneath the surface. Journal Occupational Medical, 18,165-168.

Michael, A. (1998). Stress: sinais e causas. São Paulo: Roche.

Pacheco, W., Ferreira Jr., Pereira, \& Pereira Filho. (2005). A era da tecnologia da informação e da comunicação e a saúde do trabalhador. Revista Brasileira de Medicina do Trabalho, $3(2), 114-122$.

Salanova, M. (2003). Trabajando con tecnologías y afrontando el tecnoestrés: El rol de las creencias de eficacia. Revista de Psicología del Trabajo y de las Organizaciones, 19, 225-247.

Salanova, M., Llorens, S. Cifre, E., \& Nogareda, C. (2004). Tecnoestrés: concepto, medida e intervención psicosocial. Nota técnica de prevención. Centro Nacional de Condiciones de Trabajo, Espanha.

Souto, S. M. de O. (2006). O profissional da informação frente às tecnologias do novo milênio e as exigências do mundo do trabalho. Recuperado em 16 de outubro de 2008, de http:// www.cinform.ufba.br/iv_anais/artigos/texto16.htm

Staufer, M. (1992). Technological change and the older employee: Implications for introduction and training. Behaviour and Information Technology, 11(1), 46-52.

Valle, B. de M. (1996). Tecnologia da informação no contexto organizacional. Ciências da Informação, 25(1), 5-12.

Weil, M. M., \& Rosen, L. D. (1998). Technostress: Coping with technology@work, @home, @play. New York: John Wiley and Sons.

Zauchner, S., Korunka, C., Weiss, A., \& Kafka-Lützow, A. (2000). Gender-related effects of information technology implementation. Gender, Work and Organization, 7(2), 119132. 